\title{
Los desafíos de la Nueva Ruta de la Seda para India
}

\section{Bianca Concatti}

$\mathrm{UNR}^{1}$

\section{Introducción}

El proyecto de reactivación de la Ruta de la Seda es una iniciativa que anunció el actual presidente de China, Xi Jinping, en 2013, con el objetivo de construir una red de comercio e infraestructuras para conectar Asia con Europa y África a lo largo de las antiguas rutas comerciales de la seda. Uno de los países por los que atraviesa este corredor es India, cuyo gobierno ha considerado esta iniciativa más como una amenaza que como una oportunidad, ya que afecta directamente sus intereses económicos, de seguridad nacional y su liderazgo regional. En este sentido, el objetivo de este trabajo es analizar los desafíos geopolíticos y económicos que tiene el proyecto chino de la Nueva Ruta de la Seda para la India.

El plan conocido como "la Franja y la Ruta" o "Iniciativa del Cinturón y la Ruta de la Seda" (OBOR: One Belt, One Road, por sus siglas en inglés) es una estrategia global para la cooperación económica de China en Asia, África y Europa, y en un futuro también se prevé que abarque Oceanía y América Latina. Para ello, el gigante asiático ha puesto en marcha dos corredores, uno terrestre denominado Franja Económica de la Ruta de la Seda, que está formado por cinco pasillos económicos: 1) China-Mongolia-Rusia; 2) China-Asia Central; 3) China-Pakistán; 4) China-Myanmar-Bangladesh-India y ASEAN y 5) China-Corea del Sur-Japón. Y otro marítimo, dado a conocer con el nombre de la Ruta Marítima de la Seda del siglo XXI, que se extenderá desde la costa de China hasta el Mar de China Meridional y el Estrecho de Malaca, pasando por el Océano Índico y el Golfo Pérsico hasta las aguas del Mar Mediterráneo, interconectando veinte países. Ambos proyectos se vienen desarrollando desde 2014 y su finalización está prevista para coincidir con el centenario de la República Popular de China en 2049. Además, a estas propuestas también hay que añadirle el diseño de una Ruta de la Seda Digital, que se emplazará en el espacio cibernético (Ranade, 2016).

Muchos expertos han calificado esta iniciativa como uno de los programas más ambiciosos e importantes del siglo $\mathrm{XXI}$, que se perfila para crear una arquitectura económica mundial integrada, en tanto se calcula que cuando se complete incluirá alrededor de 60 países que comprenden dos tercios de la población mundial, el 55\% del

\footnotetext{
${ }^{1}$ Estudiante avanzada de la Lic. en Relaciones Internacionales de la Facultad de Ciencia Política y Relaciones Internacionales de la Universidad Nacional de Rosario (UNR). Miembro activa hace dos años del Grupo de Estudios sobre India y del Sudeste Asiático de Rosario (GEIRSA), enmarcado en el Programa de Relaciones y Cooperación Sur-Sur (PRECSUR) de la Facultad. Trabaja e investiga la temática de China e India. Realizó un intercambio académico en la Universidad de Guangzhou, China, durante el segundo cuatrimestre del 2016. Correo electrónico: biancaconcatti@hotmail.com
} 
PIB global y el $75 \%$ de las reservas mundiales de energía. Y a esto hay que agregarle que consistirá en 900 proyectos de infraestructura, valorados en alrededor de 1,3 billones de dólares (Ranade, 2016).

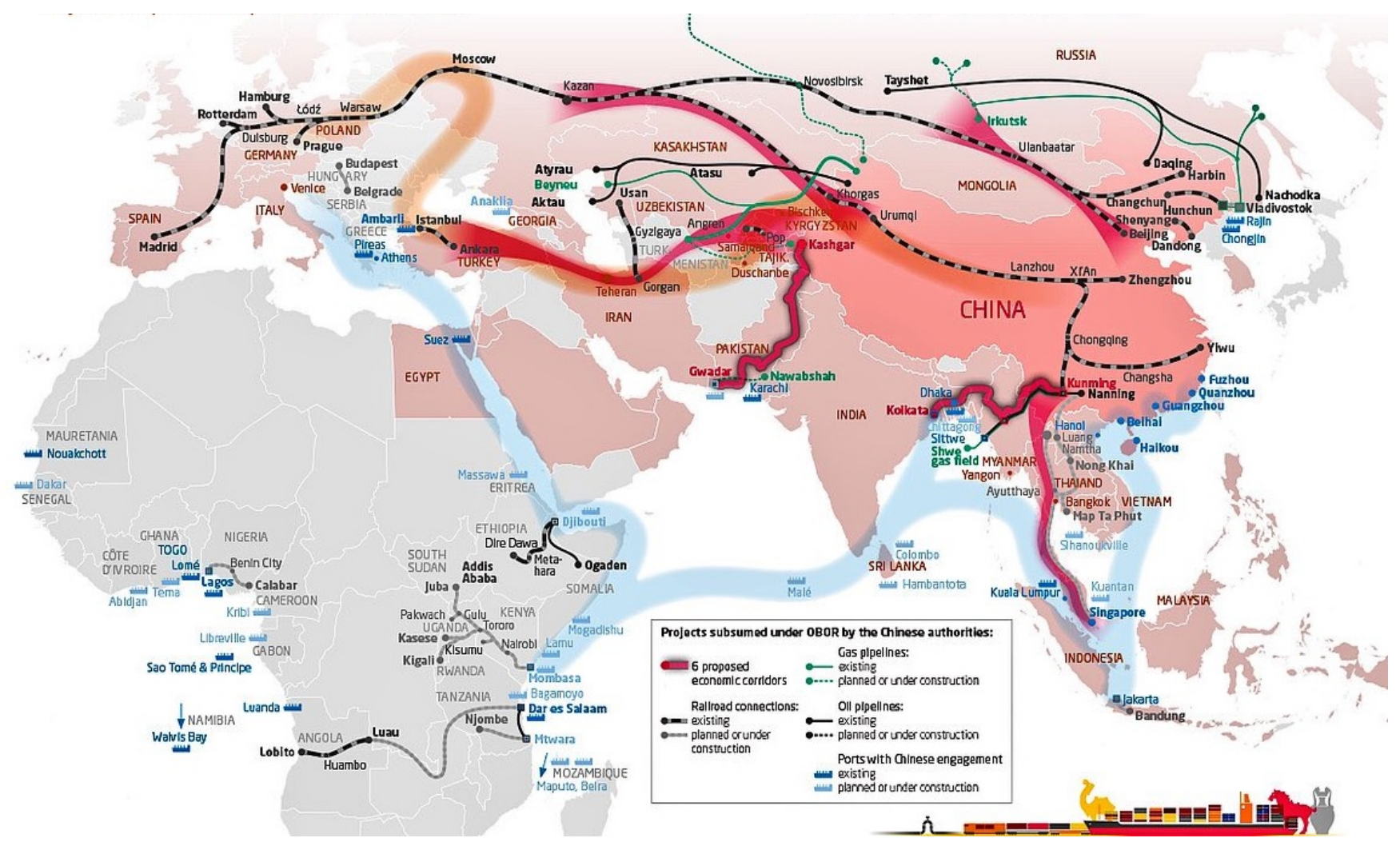

Imagen extraída dela página web de Mercator Institute for China Studies (Merics),

www.merics.org

Cabe destacar, además, que el propio gobierno chino ha resaltado que el éxito de la Franja y la Ruta dependerá del compromiso de sus participantes. Pese a ello, se han suscitado numerosos desafíos que están particularmente relacionados con complejos temas religiosos y étnicos, el terrorismo activo y el extremismo, las divisiones históricas e intereses geopolíticos en disputa. Por consiguiente, casi todos los proyectos de la Nueva Ruta de la Seda estarán imbuidos de riesgos operativos, financieros, legales y regulatorios y soberanos debido a la gran diversidad de los países involucrados y dada sus situaciones geográficas, políticas y económicas (Ahmad, 2016).

\section{Los desafíos geopolíticos}

Si bien el programa de la Franja y la Ruta ya está en marcha, algunos países han manifestado sus preocupaciones al considerar que la iniciativa es un instrumento para crear una zona terrestre y marítima contigua, cuyo objetivo es aumentar la influencia estratégica y 
económica de China en todo el mundo. Uno de los principales países que han criticado seriamente esta iniciativa ha sido la India, en tanto sus funcionarios se oponen al sostener que el proyecto sobrepone y socava la soberanía de las naciones en la medida que promueve una conectividad de manera unilateral (Mitra, 2017). Empero, el gobierno chino considera que la participación de la India en la Nueva Ruta de la Seda es clave para promover una "conectividad estratégica" y la "coordinación de políticas", por eso ha invitado al país a unirse a dos de los grandes corredores de este plan: uno es el corredor terrestre de "China-Myanmar-Bangladesh-India y ASEAN" y el otro la "Ruta Marítima de la Seda del siglo XXI" (Hong, 2016). Sin embargo, el gobierno de la India teme que esta iniciativa altere la dinámica geoestratégica y el statu quo de la región asiática, al incrementar proyectos de inversión en infraestructura en países con los que India tiene tensas relaciones, como Nepal, Sri Lanka y Pakistán.

El primer desafío geopolítico que se presenta para el país ha sido el corredor marítimo, ya que las costas indias se bañan en ambos lados por el Océano Índico, una de las zonas por donde pasa dicha franja y en donde se pretende construir una red de puertos y otras infraestructuras costeras. A través de este corredor China desea vincular los puertos del noreste, sudeste y sur de Asia con la Península Arábiga, el Golfo Pérsico, África Oriental y el Mediterráneo, formando un bucle que termina en el Pireo (Italia) y Rotterdam (Países Bajos) en Europa y Mombasa (Kenya) en África. Hay que tener en cuenta que el principal interés de Beijing reside en el mar, donde estima que es necesario sentar las bases de la seguridad y estabilidad a lo largo de la región. Cabe destacar que el gigante asiático tiene en la actualidad la industria de construcción naval más grande del mundo y ha surgido en las últimas décadas como un líder marítimo relevante. Por eso, el gobierno chino considera el Océano Índico como un espacio vital para sus intereses económicos y de seguridad en expansión, por lo que está presionando a los funcionarios indios para que se sumen a esta iniciativa. No obstante, Nueva Delhi teme perder parte de su esfera de influencia en dicho océano, por lo que aún no ha asegurado su participación en este proyecto, ya que desconfía de las intenciones estratégicas de China, que está acrecentando sustancialmente su dominación en los mares (Saran, 2015).

Ante esta perspectiva, el gobierno chino ha tratado de desviar los reclamos indios sobre la problemática en el Océano Índico, y dedica todos sus esfuerzos para convencer a la India de que se sume al Corredor de China-Myanmar-Bangladesh-India y ASEAN. Tan importantes han sido las conversaciones entre ambas partes, pero sobre todo las presiones de Beijing, que en febrero de 2017 el secretario de Relaciones Exteriores de India, Subrahmanyam Jaishankar, confirmó la participación del país en este proyecto (Time of India, 2017). Este anuncio ha significado un indudable éxito para los planes de China y un avance significativo en las relaciones bilaterales de ambos países. Sin embargo, todavía 
falta que Nueva Delhi firme oficialmente su compromiso de sumarse a dicho corredor y asegure su cooperación en el mismo. Es menester observar que el funcionario se percató de no mencionar el corredor completo, que incluye también a los países que conforman la ASEAN, por sus preocupaciones estratégicas en dicha región. Esto se debe a que en el Sudeste Asiático la India tiene un compromiso económico robusto y una participación efectiva desde hace tiempo. Si bien podría beneficiarse con los proyectos chinos, el gobierno indio estima que el objetivo de estas rutas terrestres es aumentar los enlaces de China con el mar a través de la península del sur de Asia, donde India también tiene interés de extender su liderazgo (World View, 2017).

Tanto el corredor marítimo como el terrestre a los que ha sido convocada la India dada su ubicación geográfica y su relevancia como actor económico preponderante en las relaciones asiáticas- presentan un inmenso desafío al país. Por eso, de acuerdo con algunos análisis realizados en el país, han sido considerados más como una amenaza que como una oportunidad; aunque en realidad no son estos corredores los que más preocupan a la administración del Primer Ministro indio, Narendra Modi ${ }^{2}$. Por el contrario, el "Corredor Económico China-Pakistán" es el que mayor intranquilidad viene generando al gobierno indio desde que se anunció. Este es un pasillo estratégico entre los dos países que lo componen, que endurece las relaciones entre India y su vecino pakistaní, con quien tiene una historia de conflictos y están enfrentados por cuestiones de seguridad. Según las intenciones de China, el verdadero potencial de este corredor económico radica en conectarse con la India en el este, y con Irán y las economías de Oriente Medio hacia el oeste. Para ello, el gobierno chino ha hecho una enorme apuesta de 46.000 millones de dólares para el financiamiento de una serie de proyectos energéticos, de autopistas y ferrocarriles, por lo que se calcula que cuando se completen estos planes, la infraestructura de Pakistán no sólo superará enormemente a la de la India, sino que creará una ruta alternativa para las exportaciones chinas a África y Europa (Ahmad, 2016).

El eje de esta franja estriba en el nuevo puerto pakistaní de Gwadar, en el Mar Arábigo, que será desarrollado y operado por compañías chinas que le permitirán al gigante asiático dirigir las compras de petróleo y gas desde el Golfo, y tener una presencia naval permanente en el Océano Índico occidental y una posición dominante en la desembocadura del Golfo. No queda duda de que esta dramática expansión de las fuerzas navales chinas es motivo de preocupación y alarma a largo plazo para la India (Ahmad, 2016). Sin embargo, la mayor amenaza para el gobierno indio actualmente reside en sus intereses geopolíticos de seguridad fronteriza y soberanía territorial, ya que el Corredor Económico China-Pakistán -según la administración de Modi- viola la soberanía de la India porque

\footnotetext{
${ }^{2}$ Desde 2014 en India gobierna el Bharatiya Janata Party (BJP), un partido nacionalista que por primera vez ha conformado gobierno sin la ayuda del tradicional Partido del Congreso de la India.
} 
atraviesa los territorios en disputa de Cachemira, Gilgit-Balistán y Balochistan ocupados por Pakistán. Además, según los indios este corredor se ha impuesto como una "decisión unilateral" sin consultarle al país, que asimismo genera preocupación por la falta de detalle operacional (Cai, 2017).

Otra de las cuestiones vigentes en el área está relacionada con el terrorismo activo y el extremismo, que desde la mirada de China deben ser contrarrestados con desarrollo económico. La amenaza del Movimiento Islámico del Turquestán Oriental (ETIM), formado por grupos paramilitares uigures disidentes de China, que han tomado refugio en las zonas fronterizas entre Pakistán y Afganistán y que están relacionados con Al Qaeda Talibán, ponen en peligro la ejecución de los proyectos chinos (Ahmad, 2016). La India tampoco está exenta de estas vicisitudes, lo cual es fuente de persuasión por parte de China para convencer al país de unirse al proyecto. A pesar de ello, Nueva Delhi no puede aceptar que los proyectos logísticos del Corredor Económico China-Pakistán pasen libremente por grandes partes de los territorios ocupados por Pakistán.

Un último punto que destacar en lo que refiere a los desafíos geoestratégicos tiene que ver con las relaciones sino-indias. En 1962 se desató una breve pero amarga guerra en la frontera de ambos países por conflictos territoriales, que terminó con la victoria de China. Mientras el gigante asiático considera la disputa fronteriza como un acontecimiento pasado que quiere superar lo antes posible, los indios nunca han creído que China esté dispuesta a resolver dichos problemas con el fin de cooperar con ellos en el futuro, por lo que ven sus recientes propuestas como trampas diseñadas para engañar al país a hacer concesiones de las que no podrá retractarse posteriormente (Patranobis, 2015).

\section{Los desafíos económicos}

Por su parte, en lo que atañe a cuestiones económicas, algunos académicos sostienen que la Nueva Ruta de la Seda responde profundamente a una estrategia económica por parte de China, cuya principal intención a través de estas rutas es aumentar su influencia estratégica en los países que la componen para encontrar mercados para sus empresas estatales y privadas, y con ello poder vincular sus centros de producción con los mercados y núcleos de recursos naturales de todo el mundo. En función de esto, ya han sido construidas un total de 56 zonas de cooperación económica y comercial en 20 de los países que forman la Franja y la Ruta dentro de los distintos corredores económicos. Los proyectos que se llevan a cabo en estas zonas están liderados principalmente por empresas estatales chinas y por tres grandes instituciones financieras que ejecutan los planes de inversión y financiamiento, enfocados primordialmente en la infraestructura y en la explotación de los sectores energéticos y de recursos naturales (Ferchen, 2017).

La creación de estas organizaciones financieras ha sido patrocinada por China con 
posterioridad al anuncio de la Nueva Ruta de la Seda, en conjunto con los países que considera estratégicos para este programa. Si bien dichas instituciones se plantearon en un principio con otros fines y objetivos, el gigante asiático ha redireccionado sus funciones para que formen parte esencial de los pilares económicos del OBOR. Estos organismos son el Banco Asiático de Inversión en Infraestructura (AIIB), una institución de desarrollo intergubernamental regional, cuyo propósito es apoyar la financiación de proyectos de infraestructura en la región de Asia y el Nuevo Banco de Desarrollo (NBD), antes conocido como Banco de Desarrollo de BRICS, creado por estas economías emergentes con la idea también de promover planes de infraestructura. Ambos fueron fundados en 2014 y tienen su sede central en Beijing y Shanghai respectivamente.

India es actualmente miembro permanente tanto del Banco Asiático de Inversiones en Infraestructura como del Nuevo Banco de Desarrollo, por lo que su principal desafío en este punto consiste en poder canalizar los recursos para los fines con los que dichas organizaciones se crearon y actuar de contrapeso para que China no las utilice meramente a su favor en el proyecto de la Franja y la Ruta. Adicionalmente el gobierno de Modi tiene que lidiar con las persuasiones de Beijing a través de estos dos bancos para que India se una al OBOR, en donde constantemente China resalta la gran importancia que tiene la promoción de la integración financiera. Por su parte, a fines de 2014 se creó asimismo el Fondo de la Ruta de la Seda, para los fines específicos de dicha iniciativa, en el cual China prevé destinar 40.000 millones de dólares. Empero, es preciso señalar que mientras los bancos son de carácter multilateral, el fondo es un instrumento unilateral del gobierno chino creado exclusivamente para este ambicioso programa (Ferchen, 2017).

Por otro lado, teniendo en cuenta que en los últimos años la economía de India ha crecido exponencialmente a un ritmo acelerado, se ha convertido en el único país que queda en el mundo cuya capacidad es lo suficientemente amplia para absorber la sobreproducción china de acero, cemento, plásticos e ingeniería pesada. Esto ha llevado al gigante asiático a profundizar sus relaciones comerciales con la India, para lo cual viene trabajando hace tiempo en el desarrollo y el progreso de las relaciones bilaterales en materias de interés para ambos países. Si bien en un principio los intereses de China para con la India pretendían poner freno a los Estados Unidos en su estrategia militar de AsiaPacífico, cuando la economía china empeoró en 2012, encontró una nueva y aún más urgente razón: con su enorme déficit de infraestructura y su tasa de crecimiento todavía razonablemente alta, la India era el único país que podía absorber suficientemente la producción china de maquinaria de acero y cemento, y en rigor soslayar sus dificultades en estos sectores (Jha, 2016). Sin embargo, desde la asunción del Primer Ministro indio, Narendra Modi en 2014, el eje de la política exterior de la India ha cambiado su curso y la desconfianza hacia China ha aumentado considerablemente, poniendo freno a los planes 
de Beijing en la región.

Como ya se mencionó previamente, el gobierno de Xi Jinping no ha tenido éxito al tratar de convencer a Nueva Delhi de sumarse a la Ruta Marítima de la Seda, pero sí consiguió que India se uniera al Corredor de China-Myanmar-Bangladesh-India (excluyendo a los países de la ASEAN). Aquí el gigante asiático prevé invertir 22.000 millones de dólares destinados, por una parte, a construir un puerto en Calcuta, con el cual se verá ampliamente beneficiada la India gracias a la potencial apertura económica de los estados del noreste. También existe la posibilidad de construir una red eléctrica que conectaría al Tíbet, Nepal y a la India. Además, China ha mostrado un importante interés en explotar los sectores de desarrollo ferroviario, bienes raíces, comercio electrónico y manufactura de la India. Por el momento, el gobierno indio está evaluando cuidadosamente los componentes de este corredor que puede mejorar sustancialmente su conectividad con los mercados de la región. Aunque la administración de Modi irá actuando gradualmente en los proyectos que se ejecuten en este corredor terrestre, los sectores industrial y empresarial de la India no necesitan permanecer al margen del programa de la Franja y la Ruta, dado que podrían explorar inmensas oportunidades. De hecho, ya existen empresas indias interesadas en el trabajo de proyectos y en la creación de instalaciones en los muchos parques industriales en ejecución. Algunos sectores en los que las empresas indias pueden verse beneficiadas incluyen la fabricación de bienes de ingeniería ligera y bienes de consumo, servicios profesionales, energía renovable, salud y ciencias de la vida, entre otros (Ahmad, 2016).

De todas maneras, Nueva Delhi debe analizar detalladamente esta propuesta, ya que también corre el riesgo de que los productos chinos inunden el noreste de la India, donde la escasa conectividad dificulta actualmente el acceso a los productos y personas indias. Existe el riesgo adicional de que miles de chinos se instalen ilegalmente en los escasamente poblados estados del noreste de la India, como lo han hecho en otros países. No obstante, no se puede eludir que la Franja y la Ruta reforzará el papel de China como el mayor centro de exportación de materias primas de alta gama, capital y tecnologías, relegando de esta manera sectores en los que India también tiene un fuerte potencial (Ranade, 2016).

\section{Consideraciones finales}

Para finalizar, es evidente que a pesar de ciertos beneficios económicos que pueden favorecer a la India, en verdad la Nueva Ruta de la Seda presenta mayormente un desafío, tanto geopolítico como económico para el país. Es por ello por lo que los analistas indios han considerado al OBOR más como un intento de dominación estratégica que como una agenda de cooperación económica, en tanto sospechan de las intenciones de China de acrecentar su poder marítimo en el Océano Índico y sus actividades terrestres en la región 
del sudeste asiático y de Asia meridional. En virtud de esto, Nueva Delhi ha decidido rechazar la propuesta de la Franja y la Ruta (a excepción del corredor China-MyanmarBangladesh-India que está en un proceso de revisión), ya que considera que compromete su soberanía e integridad territorial. En este sentido, ha quedado claro en el discurso de los funcionarios indios que la principal oposición de India de unirse a este proyecto tiene su razón fundamental en el conflicto latente con Pakistán por la ocupación de los territorios reivindicados indios.

Por otro lado, teniendo en cuenta las ambiciones de China, a pesar de su discurso cooperativista, intentará competir con la India por extender su influencia en diferentes sectores (geográficos, políticos, económicos, culturales e ideacionales), aunque no está claro si podrá penetrar el cerco estratégico de la India. El problema se presenta en que si la India elige permanecer fuera de la Franja y la Ruta sólo aumentará su aislamiento dentro de Asia meridional y acelerará el final de su liderazgo regional, perjudicando sus intereses geoestratégicos de seguridad nacional. Pero si no existe una verdadera coordinación y planeamiento conjunto de estos proyectos con China, corre el riesgo de convertirse por coacción o por elección en un componente subordinado de esta red dominada por China.

En conclusión, India se ve en una encrucijada ya que si rechaza unirse a este exorbitante programa le costaría aún más caro, dado que si se mantiene al margen de este proyecto le daría lugar a China de expandirse a su antojo y socavar los espacios donde la India tiene influencia, tanto a nivel geopolítico como económico. 


\section{Referencias bibliográficas}

Ahmad, T. (2016). Who's Afraid of One Belt One Road? The Wire. Recuperado de: https://thewire.in/40388/one-belt-one-road-shaping-connectivities-and-politics-in-the21st-century/

Cai, P. (2017). Why India distrusts China's One Belt One Road initiative. Lowy Institute. Recuperado de: https://www.lowyinstitute.org/the-interpreter/why-india-distrustschinas-one-belt-one-road-initiative

Ferchen, M. (2017). How China Is Using Its Economic Goals to Assert Its Global Influence. Carnegie Tsinghua. Recuperado de: http://carnegietsinghua.org/2017/05/10/howchina-is-using-its-economic-goals-to-assert-its-global-influence-pub69921?mkt_tok=eyJpljoiWldWak56SmlZemRqTVdOaylsInQiOilORGtQUzM2b3hhaF JmRjZsTzFTVIIZcjFTc1A2K3BwVmNMcGY5MTFVSHRLY3VqVVIKV3UyemNrb2xqV VJSaXAwS1dhOFwva0I1TFwvSWZJS2ImdWo2VDdpVERqQTJPNIcwTFc5d1VrVHZ RMFZXWUpOb3drK08yMWM0Q2pjcHZBRkh2In0\%3D

Hong, Z. (2016). Different Perceptions of China's One Belt One Road Initiative. IPP Review. Recuperado de http://www.ippreview.com/index.php/Blog/single/id/60.html

Jha, P. S. (2016). Why India Must Embrace China's One Belt One Road Plan. The Wire. Recuperado de: https://thewire.in/58810/india-must-embrace-chinas-one-belt-oneroad-plan/

Mitra, D. (2017). Modi Criticises China's One Belt One Road Plan, Says Connectivity Can't Undermine Sovereignty. The Wire. Recuperado de: https://thewire.in/100803/modicriticises-chinas-one-belt-one-road-plan-says-connectivity-corridors-cant-underminesovereignty/

Patranobis, S. (2015). Sushma Swaraj lays silk road for PM Modi's China visit in May. Hindustan Times. Recuperado de http://www.hindustantimes.com/india/sushmaswaraj-lays-silk-road-for-pm-modi-s-china-visit-in-may/story-

QxInDkq8pgZ6m4wsBTETsK.html

Ranade, J. (2016). Can China realise its One Belt, One Road dream?. Hindustan Times. Recuperado de http://www.hindustantimes.com/analysis/can-china-realise-its-onebelt-one-road-dream/story-Vm2Gkc2jxtwr2xqOI1BsyM.html

Saran, S. (2015). What China's One Belt and One Road Strategy Means for India, Asia and the World. The Wire. Recuperado de https://thewire.in/12532/what-chinas-one-beltand-one-road-strategy-means-for-india-asia-and-the-world/

Saran, S. (2017). China-Pakistan economic corridor violates our sovereignty, India tells Beijing. The Times of India. Recuperado de: 
http://timesofindia.indiatimes.com/india/china-pakistan-economic-corridor-violatesour-sovereignty-india-tells-beijing/articleshow/57295768.cms

Saran, S. (2017). China Paves the Way for a New Silk Road. World View. Recuperado de https://worldview.stratfor.com/article/china-paves-way-new-silk-road 